\title{
Commentary
}

\section{Behavioural variant frontotemporal dementia: At the interface of interoception, emotion and social cognition?}

\author{
Jan Van den Stock ${ }^{a, b, c, *}$ and Fiona Kumfor ${ }^{d, e, f}$ \\ a Laboratory for Translational Neuropsychiatry, Department of Neurosciences, KU Leuven, Belgium \\ ${ }^{\mathrm{b}}$ Old Age Psychiatry, University Psychiatric Center KU Leuven, Leuven, Belgium \\ c Brain and Emotion Laboratory, Department of Cognitive Neuroscience, Maastricht University, Maastricht, The \\ Netherlands \\ d School of Psychology, The University of Sydney, Sydney, NSW, Australia \\ e Brain \& Mind Centre, The University of Sydney, Sydney, NSW, Australia \\ ${ }^{\mathrm{f}}$ ARC Centre of Excellence in Cognition and Its Disorders, The University of Sydney, Sydney, NSW, Australia
}

Emerging work has begun to recognize the interaction between interoception, social cognition and emotion (Craig, 2009; Ibanez \& Manes, 2012; Shah, Catmur, \& Bird, 2017). Neurobiological theories have focused on the anterior insula as the basis of this convergence, based in part upon knowledge about the Salience Network (Seeley et al., 2007). In this commentary, we discuss the recent convergent findings reported by Adolfi et al. (2017), with a focus on how these findings can inform our conceptualization of the role of the insula across these constructs, and particularly how the neurodegenerative syndrome 'behavioral variant frontotemporal dementia' (bvFTD) can be used as a disease model for studying interactions between emotion processing, social cognition and interoception.

Adolfi et al. (2017) used a two-fold approach to study the neural crossroads of these constructs. First, they conducted three meta-analyses of functional magnetic resonance imaging (fMRI) studies in healthy adults to identify the areas that show consistent co-activation across these constructs: emotion, social cognition and interoception. A conjunction analysis across these meta-analyses revealed a set of 14 clusters in insular, frontotemporal and subcortical regions. Next, they empirically investigated emotion, social cognition and interoception in 17 stroke patients with fronto-insulotemporal damage (overlapping with regions identified by the meta-analyses). The patients displayed deficits in each of the three domains, while other cognitive abilities were relatively preserved. Hence, the authors provide converging evidence for the involvement of insular and frontotemporal regions in emotion, social cognition and interoception and propose a critical role for the insula (and associated networks) underpinning these constructs. In light of these novel findings, we consider evidence for the role of the insula across these domains in bvFTD.

\section{Behavioral variant frontotemporal dementia (bvFTD) and the anterior insula}

bvFTD is the most common variant of frontotemporal dementia (accounting for about $60 \%$ of frontotemporal dementia cases) (Johnson et al., 2005). The syndrome is characterized by progressive deterioration of behavior and social cognition, including changes in social skills, emotions, personal conduct, and self-awareness. Six core symptoms are recognized in the revised diagnostic criteria (Rascovsky et al., 2011): (i) early (i.e., within the first three years of symptom onset) behavioral disinhibition, e.g., socially inappropriate behavior, loss of manner or decorum or impulsive actions; (ii) early apathy or inertia; (iii) early loss of sympathy or empathy, e.g., diminished response to other people's needs and feelings,

\footnotetext{
* Corresponding author. Laboratory for Translational Neuropsychiatry, Department of Neurosciences, O\&N2, bus 1027, Herestraat 49, 3000 Leuven, Belgium.

E-mail address: jan.vandenstock@kuleuven.be (J. Van den Stock). 
diminished social interest; (iv) early perseverative, stereotyped or compulsive/ritualistic behavior, e.g., repetitive movements, stereotypy of speech; (v) hyperorality and dietary changes, e.g., altered food preferences, binge eating, oral exploration of inedible objects; and (vi) executive dysfunction; with at least 3 of these 6 features required for a diagnosis of bvFTD. This diagnosis is further supported by functional and/ or structural brain abnormalities (atrophy, hypometabolism or hypoperfusion) in frontal and/or anterior temporal regions, including the anterior insula (Rascovsky et al., 2011).

The insula is a structure embedded behind the lateral posterior inferior frontal lobe and anterior temporal lobe (see Fig. 1A). Typically, it is divided into anterior and posterior regions, along the central insular sulcus, with more recent work also recognizing dorsal and ventral anterior regions (Chang, Yarkoni, Khaw, \& Sanfey, 2013). The insula is highly connected, with the anterior portion connecting with the lateral orbitofrontal cortex, while the posterior portion connects with the superior temporal cortex. Of relevance here, literature linking emotion processing, social cognition and interoception with the insula, focuses predominantly on the anterior portion of this structure. In bvFTD, both the ventral (frontoinsula) and dorsal aspects of the anterior insular are affected (Seeley, 2010).

\section{Emotion processing}

Deficits in emotion recognition have been well-documented in bvFTD. While most studies have focused on verbal categorization of facial expressions (for reviews, see Kumfor, Hazelton, De Winter, Cleret de Langavant, \& Van den Stock, 2017; Kumfor \& Piguet, 2012), deficits have also been reported under different task conditions and stimulus modalities including vocal (Keane,

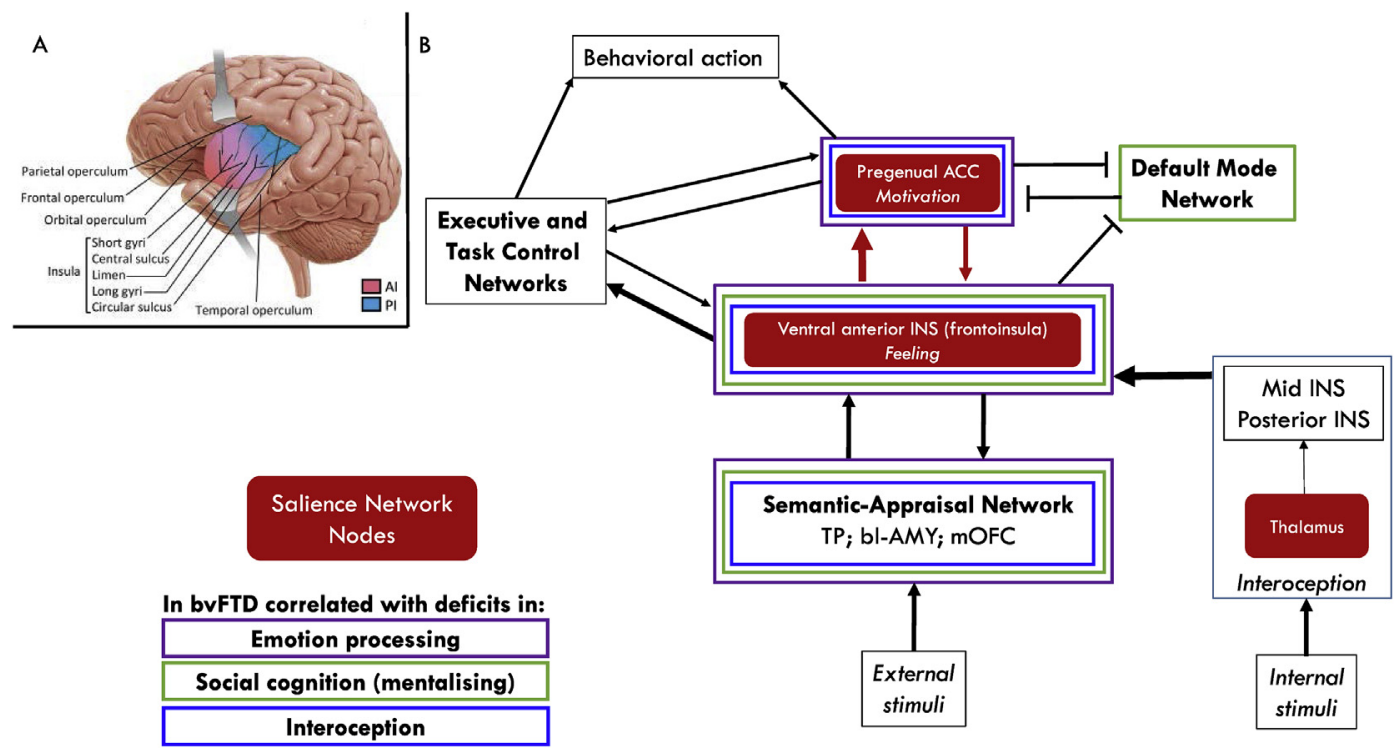

Fig. 1 - A) Anatomy of the insula. The human insular cortex is located bilaterally, deep within the lateral sulcus separating the temporal lobe from the parietal and frontal lobes. The insula is covered with folds of the adjacent frontal, parietal, and temporal opercula. The circumference of the insula is outlined by the circular sulcus, and the deep central sulcus of the insula separates the anterior and posterior parts. Three short insular gyri are found in the anterior insula (AI), whereas two long insular gyri lie in the posterior insula (PI). Cytoarchitecturally, the insula is roughly divided into anterior agranular and posterior granular sections with a transitional dysgranular mid-section. Figure reprinted from (Namkung, Kim, \& Sawa, 2017) with permission from the publisher. B) Schematic model of the salience network in relation to other large-scale brain systems and how they are associated with deficits in bvFTD in emotion processing (purple), social cognition (mentalizing) (green) and interoception (blue). bvFTD affects the salience network (printed in white against red background), initially in the central nodes, i.e., pregenual anterior cingulate cortex (pACC) and ventral anterior insula (INS). The latter processes the major ascending input streams regarding interoception (Craig, 2009). This network is closely allied with a semanticappraisal system, which includes the temporal pole (TP), medial orbitofrontal cortex (mOFC), and basolateral amygdala (blAMY), regions that construct the meaning of social and asocial stimuli in interaction with the frontoinsula and feeling states (Guo et al., 2013). The efferent salience network (pregenual ACC), triggers viscero-autonomic-emotional responses to salience and recruits executive (Seeley et al., 2007) and task control (Dosenbach et al., 2006) networks. In bvFTD, onset in the frontoinsula (FI) predicts a socially disinhibited syndrome, especially when the right FI is first affected, whereas early pregenual ACC involvement produces a more apathetic phenotype. In bvFTD, the structural integrity of areas in the Semantic-Appraisal Network is associated with deficits in emotion recognition, mentalizing and interoception (GarciaCordero et al., 2016; Kumfor et al., 2017); the fronto-insula with emotion recognition, emotion regulation, mentalizing and interoception (Garcia-Cordero et al., 2016; Guo et al., 2016; Kumfor et al., 2017); the pregenual ACC with emotion regulation and interoception (Garcia-Cordero et al., 2016; Sturm et al., 2013), and the Default Mode Network with mentalizing (Irish et al., 2012). Figure adapted from (Zhou \& Seeley, 2014) with permission from the authors and publisher. 
Calder, Hodges, \& Young, 2002; Snowden et al., 2008), bodily (Van den Stock et al., 2015) and musical (Omar et al., 2011) expressions of emotion. Emotion recognition of film stimuli is also impaired (Goodkind et al., 2015; Werner et al., 2007). Furthermore, the emotion recognition deficit in bvFTD appears to be supra-modal in nature (Keane et al., 2002; Van den Stock et al., 2015). Importantly, consistent with the results of Adolfi et al. (2017), the structural neuro-anatomy associated with impaired emotion recognition in bvFTD includes the anterior insula (Kumfor, Irish, Hodges, \& Piguet, 2013; Omar et al., 2011).

In addition to these deficits in recognizing emotions in others, experimental studies have demonstrated that bvFTD patients have abnormal emotion suppression, emotion generation and experience of self-conscious emotion. For instance, bvFTD patients viewing disgust-invoking stimuli display reduced facial expressions of disgust, reduced physiological reactivity and reduced self-reported experience of disgust, compared to controls (Eckart, Sturm, Miller, \& Levenson, 2012). Interestingly, patients with bvFTD appear to show increased reactivity to happy stimuli (films), which is related to reduced integrity of left-lateralized fronto-striatal regions, together with the left anterior insula (Sturm et al., 2015). Emotion recognition of negative film stimuli is impaired, yet the associated psychological reactivity does not appear to differ from controls (Werner et al., 2007). Future studies which incorporate simultaneous, discrete physiological measures will assist in unravelling the relationship between biological markers and behavioral performance of emotion processing in bvFTD. In addition, while previous studies commonly measure volume of large brain structures, advanced neuroimaging techniques which allow for more detailed investigation of insula integrity will help clarify the relationship between this brain region and emotion processing in bvFTD.

\section{Social cognition}

While social cognition is typically considered an umbrella term that covers several subdomains, including emotion recognition, Adolfi et al. (2017) operationalize social cognition as the interpretation of mental states of others, i.e., mentalising. While they included the terms 'empathy' and 'theory of mind' in their meta-analysis, in their lesion study they employed the 'Reading the Mind in the Eyes Test' (RMET) only (Baron-Cohen, Wheelwright, Hill, Raste, \& Plumb, 2001). On this task, participants label mental states based on visual information displayed by the eyes. The results revealed a marked impairment in the lesion group. Patients with bvFTD are also impaired on this test (Couto et al., 2013; Gregory et al., 2002; Torralva, Roca, Gleichgerrcht, Bekinschtein, \& Manes, 2009) and other tests of social cognition including both empathy and theory of mind assessment (for review see (Kumfor et al., 2017)). Importantly, again performance on such tasks is correlated with grey matter volume of the insula (e.g., Baez et al., 2017).

\section{Interoception}

Interoception refers to one's sensation of physiological body signals, including awareness of autonomic processes like one's heartbeat. In bvFTD, interoception has been elegantly investigated by Ibañez and colleagues (Garcia-Cordero et al., 2016) using a heartbeat detection task. They measured interoceptive accuracy by having participants tap on a keyboard synchronically with their heartbeat. The results revealed a marked impairment in the bvFTD group, which was neuroanatomically associated with grey matter volume in the anterior insula and fronto-temporal regions. Of relevance here, autonomic changes not related to interoception have also been reported in bvFTD, with patients showing reduced baseline cardiac vagal tone, which is related to structural and functional characteristics of the anterior insula (Guo et al., 2016).

In addition, other somatosensory deficits have also been reported in bvFTD, particularly altered noci- and thermoception. Caregiver questionnaires of sensitivity to pain and temperature have indicated alterations (both hyper- and hypo-responsiveness) in bvFTD. Converging with the results by Adolfi et al. (2017), changes in perception of pain and temperature were associated with grey matter volume of the right mid and posterior insula (Fletcher et al., 2015). Interestingly, evidence suggests a relationship between changes in autonomic functions and behavior, pointing towards a link between internal body states and social cognition. Specifically, in bvFTD, abnormal parasympathetic outflow predicts emotional traits like agreeableness (Guo et al., 2016). In keeping with this observation, when exposed to an unanticipated aversive auditory stimulus, patients with bvFTD show an intact automatic startle response, however, the subsequent self-conscious emotional response is less common in bvFTD than healthy controls (Sturm, Rosen, Allison, Miller, \& Levenson, 2006). While this study did not include neuroimaging analyses, the authors postulated that integrity of the anterior insula, together with the medial prefrontal, orbitofrontal and anterior cingulate cortices, was a plausible neural substrate, due to its role in monitoring internal physiological states (Sturm et al., 2006). Research investigating changes in interoception in these patients are only beginning to emerge; however, the results to date support Adolfi et al.'s (2017) claim that integrity of the anterior insula is essential for accurate recognition of body states and that damage to this region can give rise to changes in social behavior.

\section{Degeneration of the insula at the heart of bvFTD?}

The evidence reviewed above reveals that emotion recognition, mentalising and interoception abilities are affected in bvFTD and that each of these deficits has been independently associated with structural integrity of the insula in this population. Untangling the common mechanism underlying these apparently separable domains is challenging. The most parsimonious explanation would hypothesize an insulagrounded single common neuropsychological process underlying emotion recognition, mentalising and interoception. Currently, however, little is known about the behavioral associations between these three constructs. While increasing evidence has demonstrated a decline in emotion recognition, social cognition (mentalising) and interoception in bvFTD, 
how these constructs are related remains mostly unexplored (see Fig. 2).

Studies addressing this issue will be important to provide information about the interdependence of emotion recognition, mentalising and interoception, and how these domains relate to the anterior insula, in both normal and clinical populations. Currently, we are not aware of any paradigms that allow simultaneous assessment of these domains, but investigating interdependencies between separate tasks will help to increase our understanding of the underlying structure of these constructs. Regarding the empirical dataset of Adolfi et al. (2017), it would be interesting to investigate correlations between deficits in emotion recognition, mentalising and interoception in their patient sample, as well as exploring whether a single common factor can be extracted and related to the structural characteristics of the insula. More sophisticated mathematical procedures like structural equation modelling (e.g., path analysis) could shed light on the organizational structure of these latent variables and reveal which task domain influences the others, in certain contexts. Currently, little empirical evidence is available regarding the inter-relatedness of emotion, mentalising and interoception, as studies to date rarely investigate these domains concurrently. Clinically, each of these domains are progressively impaired in bvFTD, although it is unclear whether these domains are affected simultaneously, or if changes in one domain predicts the emergence of deficits in others. Indirect evidence suggests that mentalizing deficits may emerge in prodromal bvFTD, although this could equally be the case for emotion processing and interoception (Pardini et al., 2013). The revised diagnostic criteria further specify that socially inappropriate behavior, apathy and empathy deficits should occur within the first 3 years of symptom onset, while this is not the case for dietary changes (Rascovsky et al., 2011). If the latter reflects deficits in interoception, this may imply delayed onset of interoceptive deficits, compared to emotional and socio-cognitive abnormalities, although dietary changes may constitute a correlate of severely deteriorated interoception capacity. Furthermore, the idiosyncratic pattern of symptoms can vary significantly across bvFTD cases.

While some of the existing studies in bvFTD highlight the role of the anterior insula, many studies do not consider specific subregions of the insula. While this is in part due to the lack of specificity of available anatomical reference atlases, consideration of subregions of the insula (i.e., anterior us posterior and anterior dorsal us anterior ventral) and connections between the insula and other brain structures (e.g., orbitofrontal cortex, temporal pole, amygdala, medial prefrontal cortex) will help to shed further light on the specific role of this complex structure (Fig. 1B).

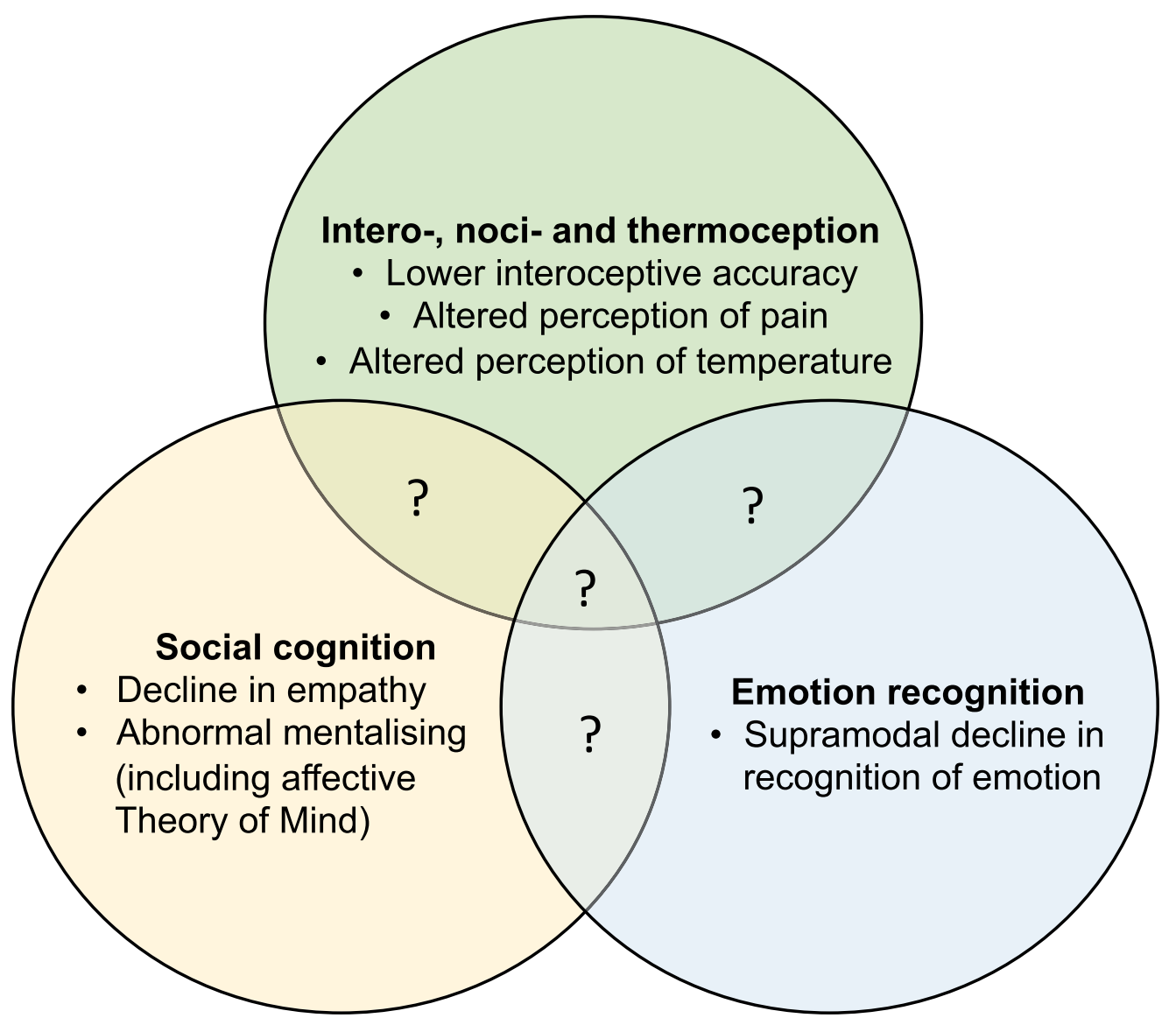

Fig. 2 - Schematic overview of the three domains investigated by Adolfi et al. (2017), integrated with associated deficits reported in bvFTD. The question marks refer to the relatively unexplored common processes underlying the three constructs of interest. 
It has also been proposed that the involvement of the anterior insula in a wide variety of neuropsychological functions reflects its primary role in saliency processing. The Salience Network, which includes the ventral anterior insula (frontoinsula) and pregenual anterior cingulate cortices is involved in processing homeostatically diverse, relevant internal and external stimuli, including emotion, pain, and reward (Seeley et al., 2007). Reduced Salience Network connectivity in bvFTD is associated with more severe clinical symptoms (Zhou et al., 2010). Functioning as an integral hub, the anterior insula also mediates dynamic interactions between other large-scale brain networks involved in other domains (Menon \& Uddin, 2010). In this context, the symptomatology observed in bvFTD may be interpreted as a failure to appropriately respond to salient cues in the environment and balance the functions between large-scale brain networks, which manifests as abnormal social behavior due to patients' inability to evaluate and infer appropriate responses in social situations (Zhou \& Seeley, 2014). Indeed, a causal influence of the Salience Network on the Default Mode Network has been linked to abnormalities in moral reasoning in bvFTD (Chiong et al., 2013). The potential of studying neuropsychiatric syndromes to shed light on brain networks is increasingly recognized (Irish, Piguet, \& Hodges, 2012; Kumfor, Dermody, \& Irish, 2015). Although only just emerging, interpretation of the role of the anterior insula from this network perspective, and its breakdown in bvFTD, is an important avenue for future work. Fig. $1 \mathrm{~B}$ provides a functional-anatomic working model of network dysfunction in bvFTD, with the anterior insula at the core (see also Zhou \& Seeley, 2014).

In conclusion, the results of Adolfi et al. (2017), converge well with the deficits observed in bvFTD and our understanding of the neurobiological basis of these changes in emotion, mentalising and interoception. A better understanding of the common factors underlying interoception, emotion recognition and mentalising will shed light on the functional properties of the anterior insula and associated regions involved in emotion, social cognition and interoception (Craig, 2009). A longitudinal approach with a protocol including tasks of emotion, social cognition and interoception, together with structural and functional brain network connectivity measures, will be important to refine socio-affective neurobiological theories and the role of the anterior insula.

\section{Funding}

JVdS is supported by Fonds Wetenschappelijk Onderzoek Vlaanderen (FWO), Alzheimer Research Foundation (P\#14013) and KU Leuven Starting Grant. This work was further supported by ForeFront, a collaborative research group dedicated to the study of frontotemporal dementia and motor neuron disease, from the National Health and Medical Research Council (NHMRC) (APP1037746) and the Australian Research Council (ARC) Centre of Excellence in Cognition and its Disorders Memory Program (CE11000102). FK is supported by an NHMRC-ARC Dementia Research Development Fellowship (APP1097026). The funding sources had no role in study design; in the collection, analysis and interpretation of data; in the writing of the report; and in the decision to submit the article for publication.

\section{R E F E R E N C E S}

Adolfi, F., Couto, B., Richter, F., Decety, J., Lopez, J., Sigman, M., et al. (2017). Convergence of interoception, emotion, and social cognition: A twofold fMRI meta-analysis and lesion approach. Cortex, 88, 124-142. http://dx.doi.org/10.1016/ j.cortex.2016.12.019.

Baez, S., Pinasco, C., Roca, M., Ferrari, J., Couto, B., GarciaCordero, I., et al. (2017). Brain structural correlates of executive and social cognition profiles in behavioral variant frontotemporal dementia and elderly bipolar disorder. Neuropsychologia. http://dx.doi.org/10.1016/ j.neuropsychologia.2017.02.012.

Baron-Cohen, S., Wheelwright, S., Hill, J., Raste, Y., \& Plumb, I. (2001). The "reading the mind in the Eyes" test revised version: A study with normal adults, and adults with asperger syndrome or high-functioning autism. Journal of Child Psychology and Psychiatry, 42(2), 241-251.

Chang, L. J., Yarkoni, T., Khaw, M. W., \& Sanfey, A. G. (2013). Decoding the role of the insula in human cognition: Functional parcellation and large-scale reverse inference. Cerebral Cortex, 23(3), 739-749. http://dx.doi.org/10.1093/cercor/bhs065.

Chiong, W., Wilson, S. M., D'Esposito, M., Kayser, A. S., Grossman, S. N., Poorzand, P., et al. (2013). The salience network causally influences default mode network activity during moral reasoning. Brain, 136(Pt 6), 1929-1941. http:// dx.doi.org/10.1093/brain/awt066.

Couto, B., Manes, F., Montanes, P., Matallana, D., Reyes, P., Velasquez, M., et al. (2013). Structural neuroimaging of social cognition in progressive non-fluent aphasia and behavioral variant of frontotemporal dementia. Frontiers in Human Neuroscience, 7, 467. http://dx.doi.org/10.3389/ fnhum.2013.00467.

Craig, A. D. (2009). How do you feel-now? The anterior insula and human awareness. Nature Reviews. Neuroscience, 10(1), 59-70. http://dx.doi.org/10.1038/nrn2555.

Dosenbach, N. U., Visscher, K. M., Palmer, E. D., Miezin, F. M., Wenger, K. K., Kang, H. C., et al. (2006). A core system for the implementation of task sets. Neuron, 50(5), 799-812. http:// dx.doi.org/10.1016/j.neuron.2006.04.031.

Eckart, J. A., Sturm, V. E., Miller, B. L., \& Levenson, R. W. (2012). Diminished disgust reactivity in behavioral variant frontotemporal dementia. Neuropsychologia, 50(5), 786-790. http://dx.doi.org/10.1016/j.neuropsychologia.2012.01.012.

Fletcher, P. D., Downey, L. E., Golden, H. L., Clark, C. N., Slattery, C. F., Paterson, R. W., et al. (2015). Pain and temperature processing in dementia: A clinical and neuroanatomical analysis. Brain, 138(Pt 11), 3360-3372. http:// dx.doi.org/10.1093/brain/awv276.

Garcia-Cordero, I., Sedeno, L., de la Fuente, L., Slachevsky, A., Forno, G., Klein, F., et al. (2016). Feeling, learning from and being aware of inner states: Interoceptive dimensions in neurodegeneration and stroke. Philosophical Transactions of the Royal Society of London. Series B, Biological Sciences, 371(1708). http://dx.doi.org/10.1098/rstb.2016.0006.

Goodkind, M. S., Sturm, V. E., Ascher, E. A., Shdo, S. M., Miller, B. L., Rankin, K. P., et al. (2015). Emotion recognition in frontotemporal dementia and Alzheimer's disease: A new film-based assessment. Emotion, 15(4), 416-427. http:// dx.doi.org/10.1037/a0039261.

Gregory, C., Lough, S., Stone, V., Erzinclioglu, S., Martin, L., BaronCohen, S., et al. (2002). Theory of mind in patients with frontal variant frontotemporal dementia and Alzheimer's disease: 
Theoretical and practical implications. Brain, 125(Pt 4), 752-764.

Guo, C. C., Gorno-Tempini, M. L., Gesierich, B., Henry, M., Trujillo, A., Shany-Ur, T., et al. (2013). Anterior temporal lobe degeneration produces widespread network-driven dysfunction. Brain, 136(Pt 10), 2979-2991. http://dx.doi.org/ 10.1093/brain/awt222.

Guo, C. C., Sturm, V. E., Zhou, J., Gennatas, E. D., Trujillo, A. J., Hua, A. Y., et al. (2016). Dominant hemisphere lateralization of cortical parasympathetic control as revealed by frontotemporal dementia. Proceedings of the National Academy of Sciences of the United States of America, 113(17), E2430-E2439. http://dx.doi.org/10.1073/pnas.1509184113.

Ibanez, A., \& Manes, F. (2012). Contextual social cognition and the behavioral variant of frontotemporal dementia. Neurology, 78(17), 1354-1362. http://dx.doi.org/10.1212/ WNL.0b013e3182518375.

Irish, M., Piguet, O., \& Hodges, J. R. (2012). Self-projection and the default network in frontotemporal dementia. Nature Reviews. Neurology, 8(3), 152-161. http://dx.doi.org/10.1038/ nrneurol.2012.11.

Johnson, J. K., Diehl, J., Mendez, M. F., Neuhaus, J., Shapira, J. S., Forman, M., et al. (2005). Frontotemporal lobar degeneration: Demographic characteristics of 353 patients. Archives of Neurology, 62(6), 925-930. http://dx.doi.org/10.1001/ archneur.62.6.925.

Keane, J., Calder, A. J., Hodges, J. R., \& Young, A. W. (2002). Face and emotion processing in frontal variant frontotemporal dementia. Neuropsychologia, 40(6), 655-665.

Kumfor, F., Dermody, N., \& Irish, M. (2015). Considering the impact of large-scale network interactions on cognitive control. The Journal of Neuroscience, 35(1), 1-3. http://dx.doi.org/ 10.1523/JNEUROSCI.4213-14.2015.

Kumfor, F., Hazelton, J., De Winter, F. L., Cleret de Langavant, L., \& Van den Stock, J. (2017). Clinical studies of social neuroscience: A lesion model approach. In A. Ibanez, L. Sedeno, \&

A. M. Garcia (Eds.), Neuroscience and Social Science: The Missing Link. Springer.

Kumfor, F., Irish, M., Hodges, J. R., \& Piguet, O. (2013). Discrete neural correlates for the recognition of negative Emotions: Insights from frontotemporal dementia. Plos One, 8(6), e67457. http://dx.doi.org/10.1371/journal.pone.0067457.

Kumfor, F., \& Piguet, O. (2012). Disturbance of emotion processing in frontotemporal dementia: A synthesis of cognitive and neuroimaging findings. Neuropsychology Review, 22(3), 280-297. http://dx.doi.org/10.1007/s11065-012-9201-6.

Menon, V., \& Uddin, L. Q. (2010). Saliency, switching, attention and control: A network model of insula function. Brain Structure \& Function, 214(5-6), 655-667. http://dx.doi.org/ 10.1007/s00429-010-0262-0.

Namkung, H., Kim, S. H., \& Sawa, A. (2017). The Insula: An underestimated brain area in clinical neuroscience, psychiatry, and neurology. Trends in Neurosciences, 40(4), 200-207. http://dx.doi.org/10.1016/j.tins.2017.02.002.

Omar, R., Henley, S. M., Bartlett, J. W., Hailstone, J. C., Gordon, E., Sauter, D. A., et al. (2011). The structural neuroanatomy of music emotion recognition: Evidence from frontotemporal lobar degeneration. NeuroImage, 56(3), 1814-1821. http:// dx.doi.org/10.1016/j.neuroimage.2011.03.002.

Pardini, M., Emberti Gialloreti, L., Mascolo, M., Benassi, F., Abate, L., Guida, S., et al. (2013). Isolated theory of mind deficits and risk for frontotemporal dementia: A longitudinal pilot study. Journal of Neurology, Neurosurgery \& Psychiatry, 84(7), 818-821. http://dx.doi.org/10.1136/jnnp-2012-303684.
Rascovsky, K., Hodges, J. R., Knopman, D., Mendez, M. F., Kramer, J. H., Neuhaus, J., et al. (2011). Sensitivity of revised diagnostic criteria for the behavioural variant of frontotemporal dementia. Brain, 134(Pt 9), 2456-2477. http:// dx.doi.org/10.1093/brain/awr179.

Seeley, W. W. (2010). Anterior insula degeneration in frontotemporal dementia. Brain Structure \& Function, 214(5-6), 465-475. http://dx.doi.org/10.1007/s00429-010-0263-z.

Seeley, W. W., Menon, V., Schatzberg, A. F., Keller, J., Glover, G. H., Kenna, H., et al. (2007). Dissociable intrinsic connectivity networks for salience processing and executive control. The Journal of Neuroscience, 27(9), 2349-2356. http://dx.doi.org/ 10.1523/JNEUROSCI.5587-06.2007.

Shah, P., Catmur, C., \& Bird, G. (2017). From heart to mind: Linking interoception, emotion, and theory of mind. Cortex. http:// dx.doi.org/10.1016/j.cortex.2017.02.010.

Snowden, J. S., Austin, N. A., Sembi, S., Thompson, J. C., Craufurd, D., \& Neary, D. (2008). Emotion recognition in Huntington's disease and frontotemporal dementia. Neuropsychologia, 46(11), 2638-2649. http://dx.doi.org/10.1016/ j.neuropsychologia.2008.04.018.

Sturm, V. E., Rosen, H. J., Allison, S., Miller, B. L., \& Levenson, R. W. (2006). Self-conscious emotion deficits in frontotemporal lobar degeneration. Brain, 129(Pt 9), 2508-2516. http://dx.doi.org/ 10.1093/brain/awl145.

Sturm, V. E., Sollberger, M., Seeley, W. W., Rankin, K. P., Ascher, E. A., Rosen, H. J., et al. (2013). Role of right pregenual anterior cingulate cortex in self-conscious emotional reactivity. Social Cognitive and Affective Neuroscience, 8(4), 468-474. http://dx.doi.org/10.1093/scan/nss023.

Sturm, V. E., Yokoyama, J. S., Eckart, J. A., Zakrzewski, J., Rosen, H. J., Miller, B. L., et al. (2015). Damage to left frontal regulatory circuits produces greater positive emotional reactivity in frontotemporal dementia. Cortex, 64, 55-67. http://dx.doi.org/10.1016/j.cortex.2014.10.002.

Torralva, T., Roca, M., Gleichgerrcht, E., Bekinschtein, T., \& Manes, F. (2009). A neuropsychological battery to detect specific executive and social cognitive impairments in early frontotemporal dementia. Brain, 132(Pt 5), 1299-1309. http:// dx.doi.org/10.1093/brain/awp041.

Van den Stock, J., De Winter, F. L., de Gelder, B., Rangarajan, J. R., Cypers, G., \& et al.Maes, F., et al. (2015). Impaired recognition of body expressions in the behavioral variant of frontotemporal dementia. Neuropsychologia, 75, 496-504. http://dx.doi.org/10.1016/j.neuropsychologia.2015.06.035.

Werner, K. H., Roberts, N. A., Rosen, H. J., Dean, D. L., Kramer, J. H., Weiner, M. W., et al. (2007). Emotional reactivity and emotion recognition in frontotemporal lobar degeneration. Neurology, 69(2), 148-155. http://dx.doi.org/ 10.1212/01.wnl.0000265589.32060.d3.

Zhou, J., Greicius, M. D., Gennatas, E. D., Growdon, M. E., Jang, J. Y., Rabinovici, G. D., et al. (2010). Divergent network connectivity changes in behavioural variant frontotemporal dementia and Alzheimer's disease. Brain, 133(Pt 5), 1352-1367. http://dx.doi.org/10.1093/brain/awq075.

Zhou, J., \& Seeley, W. W. (2014). Network dysfunction in Alzheimer's disease and frontotemporal dementia: Implications for psychiatry. Biological Psychiatry, 75(7), 565-573. http://dx.doi.org/10.1016/j.biopsych.2014.01.020.

Received 17 May 2017

Revised 18 July 2017

Accepted 1 August 2017 